\title{
Capturing Multicellular System Designs Using Synthetic Biology Open Language (SBOL)
}

Bradley Brown, ${ }^{\dagger}$ Bryan Bartley, Jacob Beal, Jasmine E. Bird," Ángel GoñiMoreno, ${ }^{\ddagger}$ James Alastair McLaughlin, ${ }^{\ddagger}$ Göksel Mısırlı, ${ }^{\S}$ Nicholas Roehner, David James Skelton, ${ }^{\ddagger}$ Chueh Loo Poh, ${ }^{\square}$ Irina Dana Ofiteru, ${ }^{\dagger}$ Katherine James ${ }^{b}$ and Anil Wipat*,‡

† School of Engineering, Newcastle University, NE1 TRU, United Kingdom

// Raytheon BBN Technologies, Cambridge, MA, 02138, United States

II School of Natural and Environmental Sciences, Newcastle University, NE1 7RU United Kingdom

\# School of Computing, Newcastle University, NE4 5TG, United Kingdom

\# Centro de Biotecnología y Genómica de Plantas (CBGP, UPM-INIA), Universidad Politénica de Madrid (UPM) - Instituto Nacional de Investigación y Tecnología Agraria y Alimentaria (INIA) Campus de Montegancedo-UPM, 28223 Pozuelo de Alarcon, Madrid, Spain

§School of Computing and Mathematics, Keele University, ST5 5BG, United Kingdom

[ Department of Biomedical Engineering and NUS Synthetic Biology for Clinical and Technological Innovation (SynCTI), National University of Singapore, Singapore

b Department of Applied Sciences, Northumbria University, United Kingdom

*Email: anil.wipat@newcastle.ac.uk 


\section{Supplementary Images}

A)

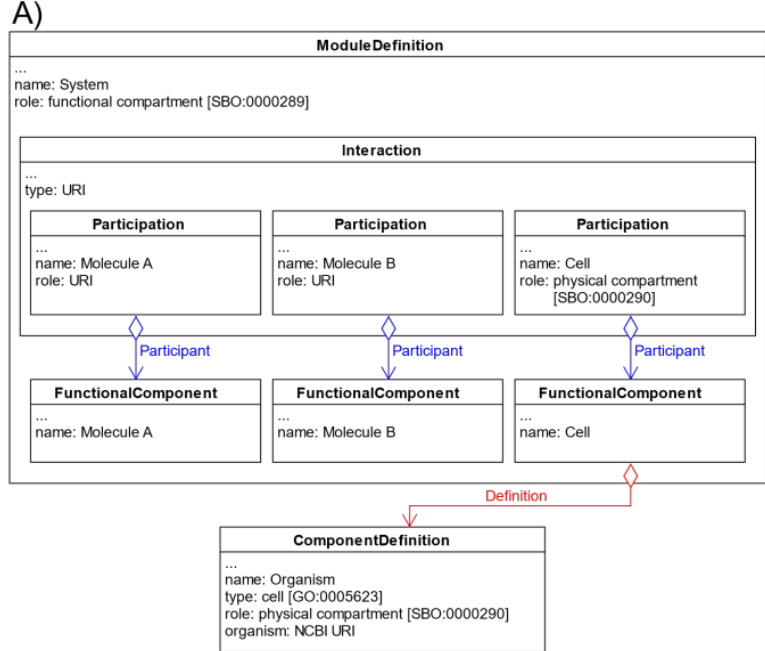

B)

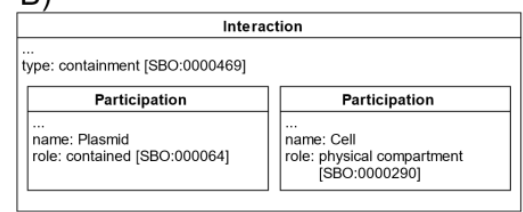

Supplementary Figure 1. Uniform Markup Language (UML) Diagram Depicting a Representation of Cells Using Existing SBOL Classes. (A) Recommended approach for representing cells in SBOL. Information about a type of cell is captured using the ComponentDefinition class annotated with a URI pointing to an entry in the NCBI Taxonomy database. When used to capture information about a cell, the ComponentDefinition class should have a type of 'Cell' from the Gene Ontology (GO), and an SBO role of 'physical compartment'. The ModuleDefinition class is used to represent a system that involves the cell defined by the ComponentDefinition class. Various system-wide components, including the cell, are included in the design as FunctionalComponents, and processes are captured using the Interaction class. Processes that are contained within the cell are represented by including the cell as a participant with a role of 'physical compartment'. (B) The Interaction class can also be used to explicitly state that an entity is contained inside a cell. In this example, a plasmid is specified as being contained within the cell. 


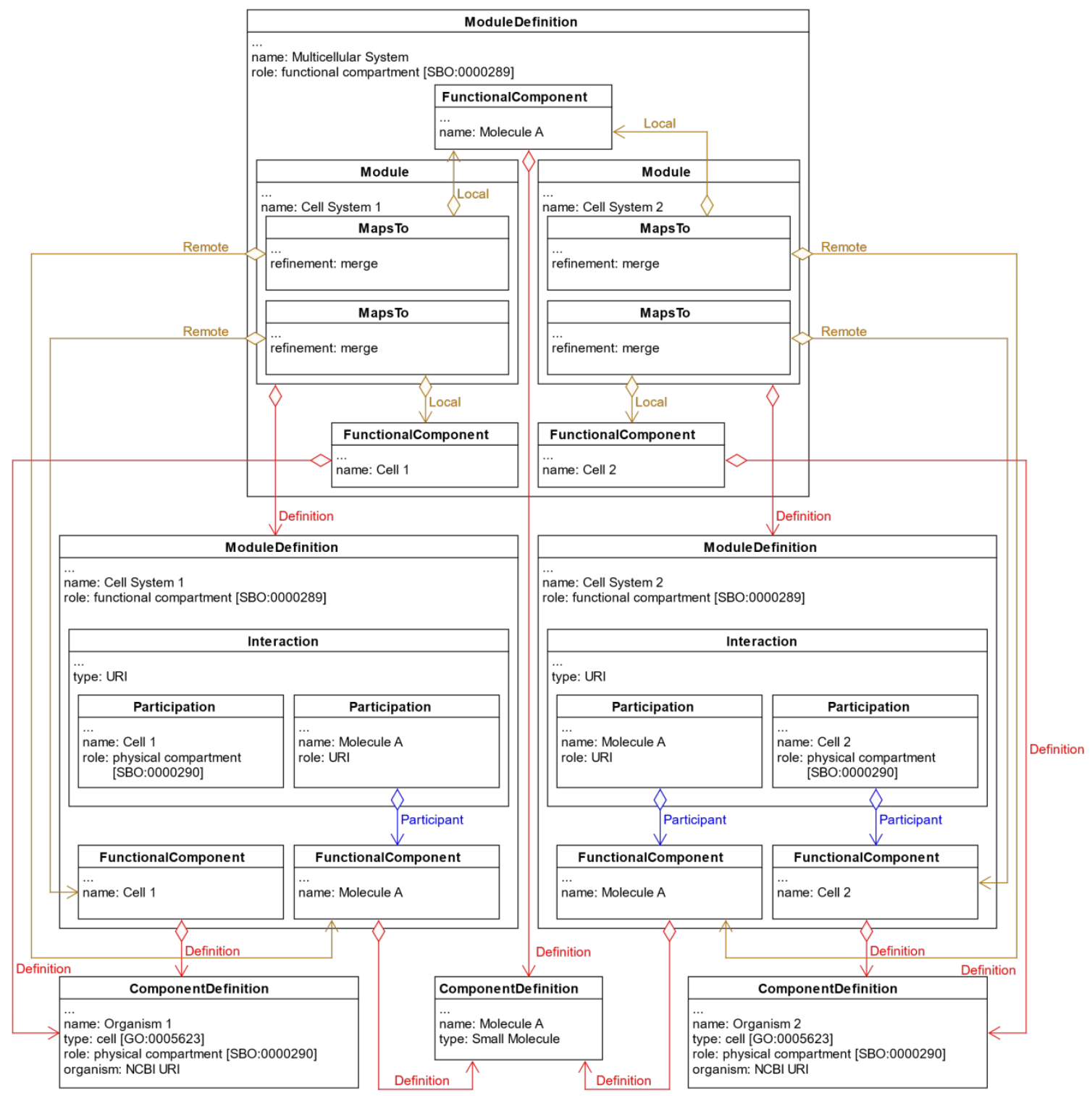

Supplementary Figure 2. UML Diagram Depicting the Composition of a Multicellular Design from Designs with Single Types of Cell. Shown here is a design involving two cell types that both interact with the small molecule 'Molecule A'. Designs for Cell 1 and Cell 2 are captured using the approach depicted in Figure 1a. The overall multicellular system is represented by a ModuleDefinition with a role of 'functional compartment', which is an SBO term. The two systems involving Cell 1 and Cell 2 are included in this multicellular design as instances of the Module class. Entities in the multicellular design, including those in the Cell 1 and Cell 2 system designs, are instantiated as a FunctionalComponent. Instances of the MapsTo class are used to map entities specified in both the individual cell systems, and the multicellular system. 


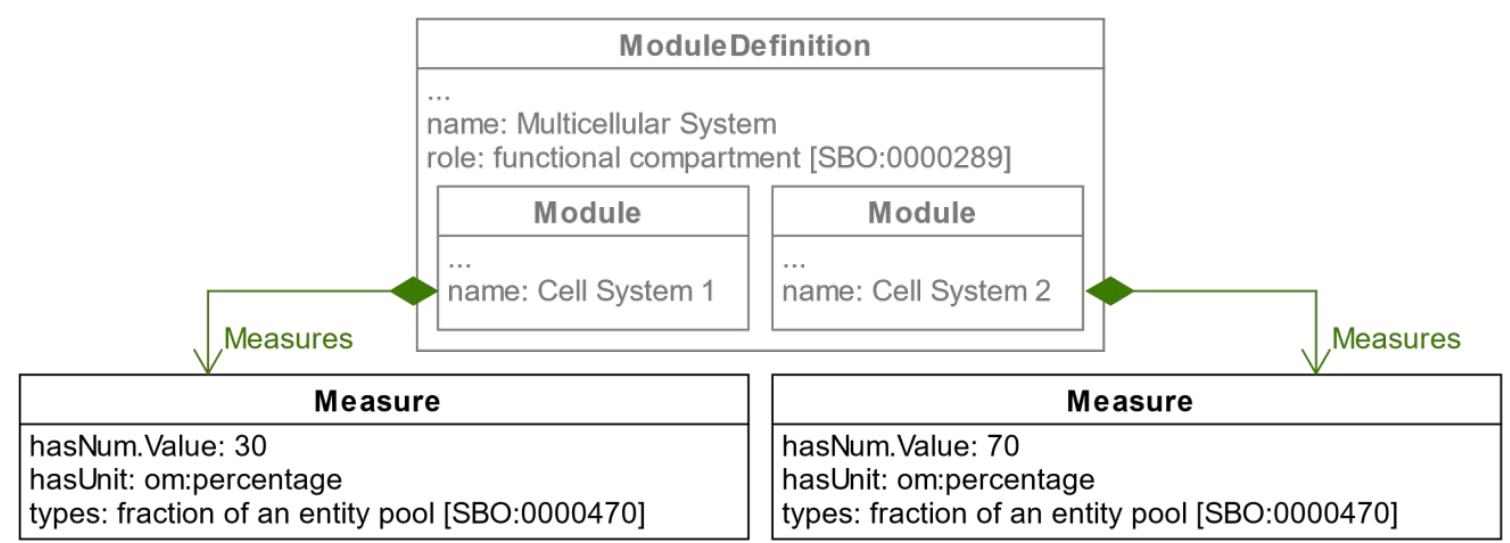

Supplementary Figure 3. UML Diagram Depicting the Representation of Cell Proportions in a Design. The ratios of cell types in a multicellular system can be representing using instances of the Measure class in SBOL 2.3 Error! Reference source not found. The proportion of cells can be defined using any appropriate unit of measurement from the Ontology of Units of Measure and Related Concepts (OM) Error! Reference source not found. In this example, an instance of the Measure class is used to annotate Module instances representing cell systems in the multicellular design; cell system 1 comprises $30 \%$ of the overall cell population, and cell system 2 comprises the remaining $70 \%$. 


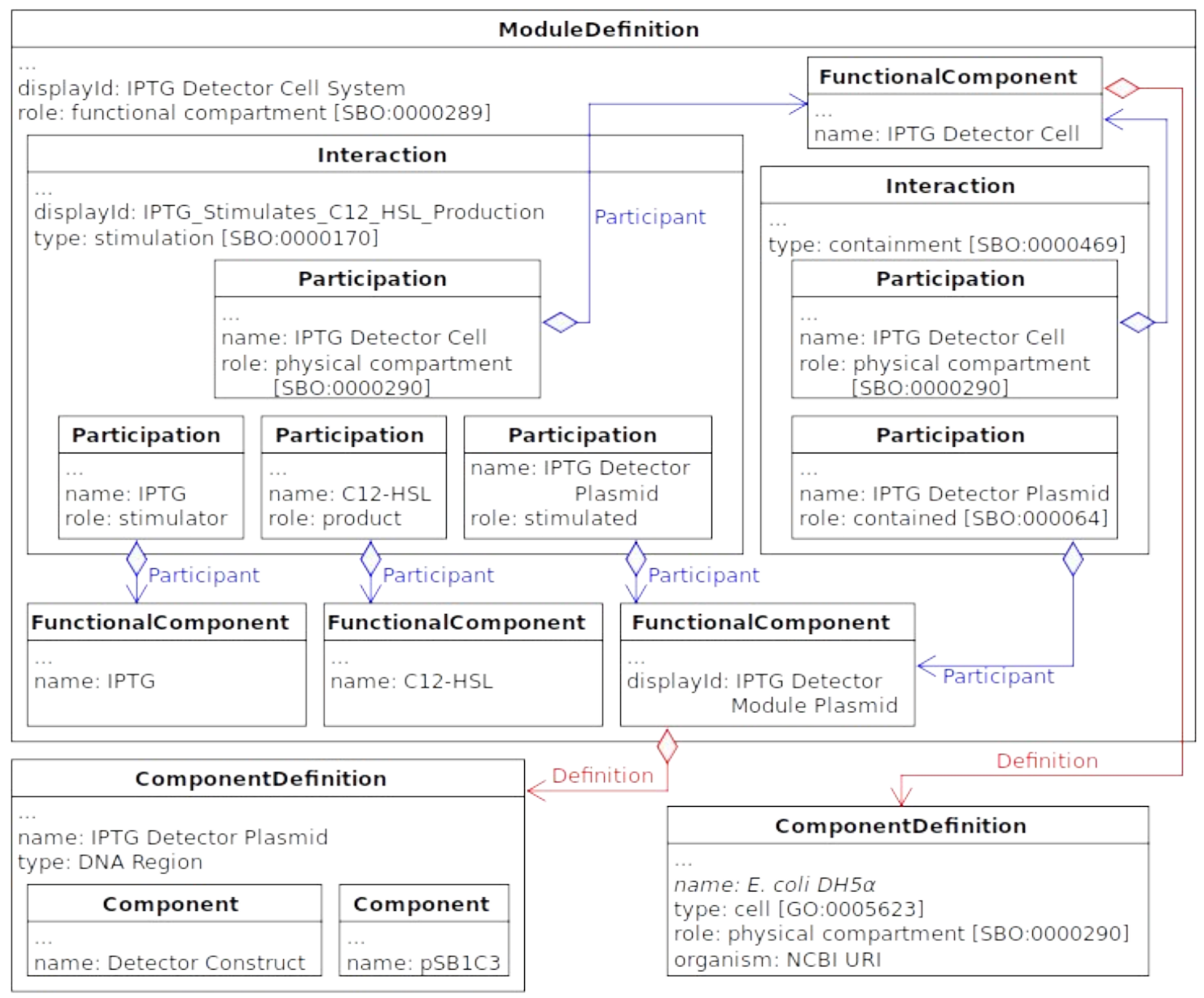

Supplementary Figure 4. Capturing the Isopropyl $\beta$ - d-1-thiogalactopyranoside (IPTG) Detector Cell Design from the Modular, Multicellular Biosensor using SBOL. The cell strain used in this design is E. coli DH5 $\alpha$, which is entry 668369 in the NCBI Taxonomy Database. This cell contains the IPTG Detector Module Plasmid, which allows the cell to produce the small molecule C12-HSL in the presence of the small molecule IPTG. An instance of the Interaction class is used to explicitly capture the containment of the DNA plasmid by the cell. 


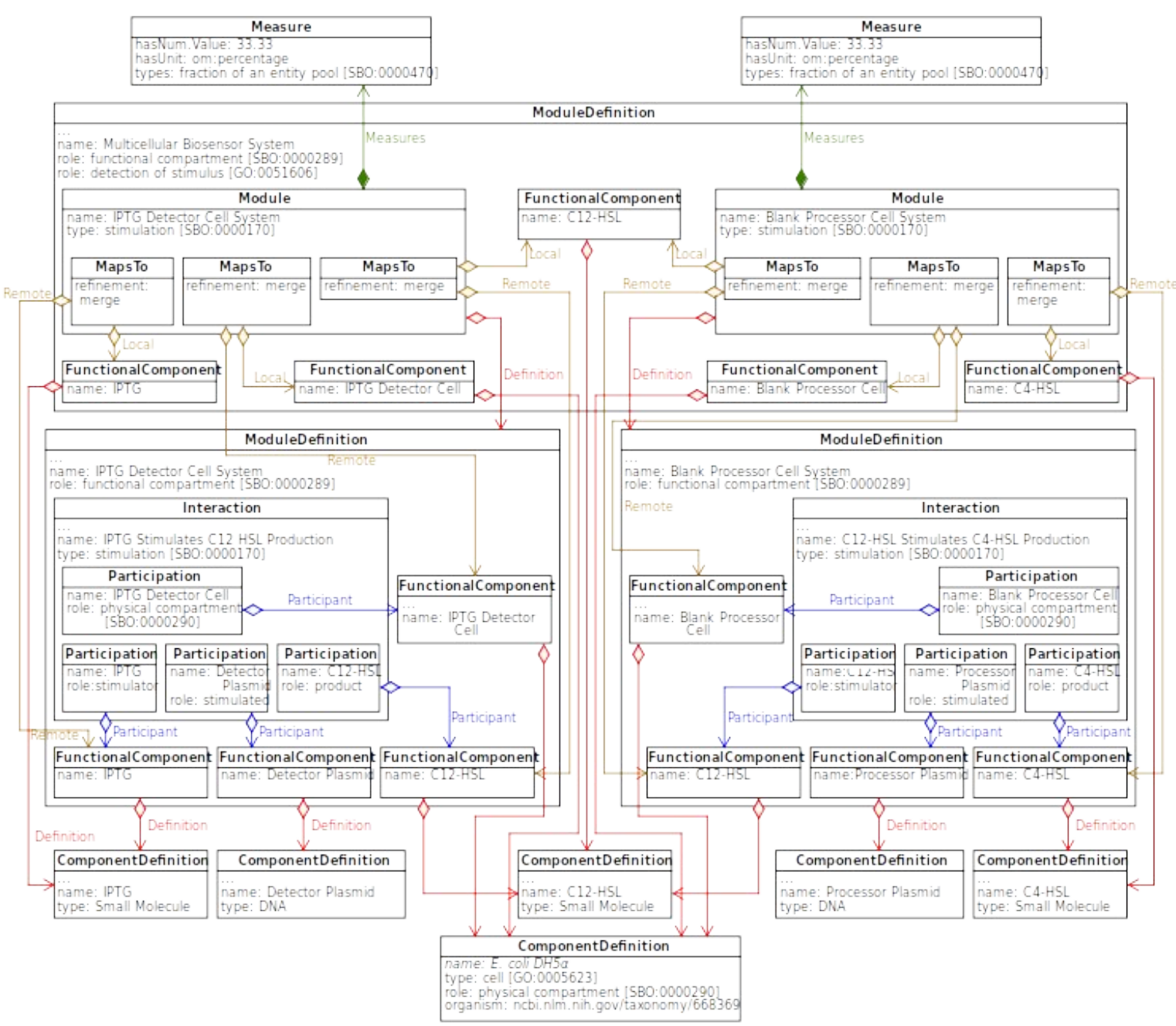

Supplementary Figure 5. Capturing Interactions between the IPTG Detector and Blank Processor Cells using SBOL. The Detector Cell produces C12-HSL in the presence of IPTG, and the Processor Cell produces C4-HSL in the presence of C12-HSL. This approach explicitly captures that the C12-HSL used in both cell designs is the same molecule since both instances of C12-HSL are defined by the same ComponentDefintion. Two variants of the $E$. coli $\mathrm{DH} 5 \alpha$ strain are included in the same multicellular system design as a FunctionalComponent and labelled as an IPTG Detector Cell and Blank Processor Cell. Additionally, all of the important entities are included as instances of the FunctionalComponent class. The two system designs involving the Detector and Processor Cells are also instantiated as a Module. Instances of the MapsTo class are used to link identical entities in both the multicellular and unicellular designs. For example, the IPTG small molecule in the multicellular design is linked to the IPTG molecule in the Detector Cell system design via the MapsTo class, indicating that they are the same molecule. 


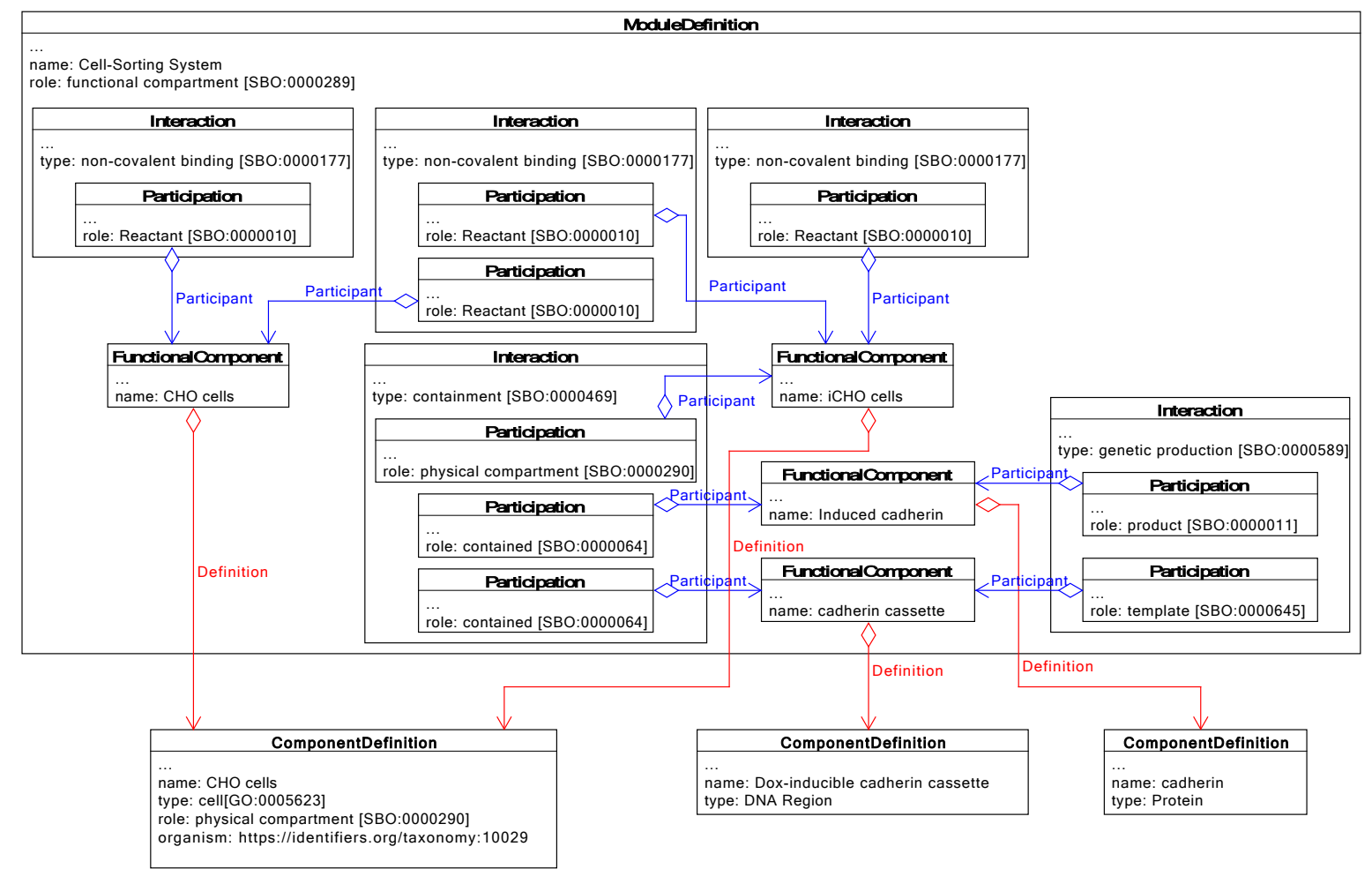

Supplementary Figure 6. Capturing Information about a Cell Sorting System using SBOL. Two cell types are captured in this system, both of which are $\mathrm{CHO}$ cells. These two cell types are instantiated in a ModuleDefinition representing the entire system, and the Interaction class is used to define their functions, and hence the functionality of the system as a whole. 